\title{
A stochastic model updating method for parameter variability quantification based on response surface models and Monte Carlo simulation
}

\author{
Sheng-En Fang Wei-Xin Ren Ricardo Perera
}

\begin{abstract}
A B S T R A C T
Stochastic model updating must be considered for quantifying uncertainties inherently existing in real-world engineering structures. By this means the statistical properties, instead of deterministic values, of structural parameters can be sought indicating the parameter variability. However, the implementation of stochastic model updating is much more complicated than that of deterministic methods particularly in the aspects of theoretical complexity and low computational efficiency. This study attempts to propose a simple and cost-efficient method by decomposing a stochastic updating process into a series of deterministic ones with the aid of response surface models and Monte Carlo simulation. The response surface models are used as surrogates for original FE models in the interest of programming simplification, fast response computation and easy inverse optimization. Monte Carlo simulation is adopted for generating samples from the assumed or measured probability distributions of responses. Each sample corresponds to an individual deterministic inverse process predicting the deterministic values of parameters. Then the parameter means and variances can be statistically estimated based on all the parameter predictions by running all the samples. Meanwhile, the analysis of variance approach is employed for the evaluation of parameter variability significance. The proposed method has been demonstrated firstly on a numerical beam and then a set of nominally identical steel plates tested in the laboratory. It is found that compared with the existing stochastic model updating methods, the proposed method presents similar accuracy while its primary merits consist in its simple implementation and cost efficiency in response computation and inverse optimization.
\end{abstract}

\section{Introduction}

FE model updating methods have been extensively investigated in recent years $[1,2]$ and used for damage identification purposes [3-7]. However, almost all the existing methods are deterministic since the presence of uncertainties in parameters and measurements are not taken into account. For real-world engineering structures, such simplification is 
generally impractical and thus highly limits the applications of deterministic model updating methods. Therefore, stochastic updating methods involving uncertainty analysis are of great importance from a practical point of view. In a broad sense, uncertainties can be classified into two categories of aleatory (irreducible) and epistemic (reducible) uncertainties [8] and in many cases there is no strict distinction between these two categories. Aleatory uncertainty may result from geometric dimension variability due to manufacturing tolerances or inherent variability of materials such as concrete, while epistemic uncertainty is caused by lack of knowledge (e.g. due to limited experimental data). In mechanical and civil engineering aspects, aleatory uncertainty mainly refers to parameter variability in both geometry and materials, which is of primary concern in the early stage of stochastic model updating (SMU) research. An SMU procedure aiming at quantifying such variability should be implemented in order to determine the statistical properties, instead of deterministic values, of parameters [9-17]. Meanwhile, according to the preknowledge of the probability distributions of parameter variability, different methodologies such as probabilistic methods, fuzzy logic methods and interval analysis [18] can be adopted for uncertainty propagation and quantification. In the aspect of probabilistic SMU, the early work can be traced back to that of Collins [9], in which a model updating procedure is developed considering the uncertain parameters having normal probabilistic distributions. The measurement errors were also involved in the analysis by assuming the uncertainty as being independent of the parameter variability. The method was successfully performed on a simulated beam and a tested Saturn V vibration model. Friswell [10] proposed a minimum variance estimator for parameter adjustment using a model updating strategy. Different to Ref. [9], both parameter variability and measurement errors were considered to be correlated. Faster convergence speed and higher accuracy in parameter identification were achieved after validating the algorithm on some numerical examples. More recently, Beck and Katafygiotis [11] presented a Bayesian statistical framework for quantitatively analyzing the prediction accuracy of dynamic loadings. The analysis focused on the mismatch between the detailed FE model providing the uncertain accuracy in the predicted response and the incomplete and noisy test data. The accuracy problem was caused by the modeling errors resulting from such as material variability and the lack of knowledge of boundary conditions. Then the probability models were established and updated using the Bayes' theorem in order to quantify the modeling uncertainties. Steenackers and Guillaume [12] extended the traditional deterministic FE model updating technique to a stochastic one by considering the measurement statistics as an inverse weighting factor in the cost function to be minimized. The frequency standard deviations were obtained by repeating the modal testing on a single plate whose length, width and Young's modulus were updated. Mares and his colleagues [13,14] established a Monte Carlo (MC) based inverse problem and the measurements from a set of seemingly identical structures were used to identify the parameter variability. The discrepancies between the means of the predicted and measured frequencies were minimized by a gradient-andregression method. Recently, perturbation methods have been effectively employed in SMU [15-17] in which random variable vectors are used as the perturbation to parameter means in model updating equations including deterministic terms. The establishment of an inverse optimization problem is still complicated but is more cost-efficient than an MC based SMU method. For forward uncertainty propagation in perturbation based SMU methods, MC simulation (MCS), the meancentered first-order perturbation method and the asymptotic integral method [17,19] can be used, among which MCS may provide most accurate estimation and then the asymptotic integral method. One point should be taken care that the estimation error of response statistics must be significantly lower than the optimization convergence requirement [15], which might result in considerable computational costs. Although perturbation methods may provide satisfactory predictions, their limitations lie in the assumptions of small uncertainties and normal probability distributions of parameters. Meanwhile, presently for most SMU methods, parameter means can be well predicted, while variance (covariance matrix) predictions highly depart from true values. To partly overcome this drawback, an SMU procedure can be divided into two independent steps by which means the parameter means and covariance matrices are updated in sequence [20].

It has been found that MC based SMU methods can provide accurate predictions but require heavy computation. At the same time, Bayesian or perturbation methods are complicated in their implementation and also have some limitations. Therefore, this paper proposes a new SMU method in possession of theoretical simplification, cost efficiency in updating and easy implementation. Forward uncertainty propagation is not required highly simplifying the updating procedure. The method adopts MCS for response sample generation and also employs response surface (RS) models for the purpose of fast response computation and easy inverse optimization. The feasibility and accuracy of the proposed method has been validated using a numerical beam and then a set of tested plates. Moreover, an uncertainty significance evaluation method is also developed based on the theory of analysis of variance (ANOVA) [21,22], which can effectively analyze the uncertainty effects of parameters on responses.

\section{Theory}

\subsection{Response surface models}

The FE models of complex structures are usually very complex and their inverse optimization usually requires heavy computational expense with high possibility of failure due to ill-conditioned sensitivity matrices. Therefore, one may seek for other physical or mathematical models to substitute for the FE models for establishing a healthy and cost-efficient optimization problem. Neural networks have been found to be an option [23] but the requirement of training samples for a qualified simulation highly increases computational cost. And the black-box structure of a network may limit its further 
application with other programs. Lately, an alternative meta-model type called response surfaces has been employed in the FE model updating realm $[6,7,24,25]$. Compared with traditional sensitivity based FE model updating methods, the easy implementation and high computational efficiency of RS based model updating methods have been well approved in the literature.

An RS model is in essence an explicit mathematical expression correlating the parameter $\xi$ of a physical system with the response $y[26]$ :

$$
y=f\left(\xi_{1}, \xi_{2}, \ldots, \xi_{k}\right)+\varepsilon
$$

where $f$ denotes the mapping function; $\varepsilon$ denotes the sources of variability which cannot be taken into account in $f ; k$ is the number of the parameters. Practically, $\xi$ is coded into a dimensionless variable (vector) $x$ having mean zero and the same standard deviation of $\xi$ :

$$
y=f\left(x_{1}, x_{2}, \ldots, x_{k}\right)+\varepsilon=\beta_{0}+\sum_{i=1}^{k} \beta_{i} x_{i}+\sum_{i=1}^{k} \beta_{i i} x_{i}^{2}+\sum_{i<j} \sum_{j=2}^{k} \beta_{i j} x_{i} x_{j}+\varepsilon
$$

which represents a second-order polynomial expression adequate for most engineering problems. Here $\beta$ denotes the partial regression coefficients to be determined by least squares estimation of $\varepsilon ; x_{i}$ represents the main effect of parameter $i$ and $x_{i} x_{j}$ gives the interaction effect between two parameters. The terms in Eq. (2) are not unalterable. For example, the interaction terms can be neglected if they do not show significant effects on the response. On the other hand, higher order terms can also be added for a more flexible model. Then using statistical regression, the estimated response $\hat{y}$ can be given as

$$
\hat{y}=b_{0}+\sum_{i=1}^{k} b_{i} x_{i}+\sum_{i=1}^{k} b_{i i} x_{i}^{2}+\sum_{i<j} \sum_{j=2}^{k} b_{i j} x_{i} x_{j}
$$

where $b$ is the unbiased estimated value of $\beta$.

In practice, an RS should fit the samples in the parameter design space and these samples are designed by using the design of experiment theory [22]. A common and useful design for a second-order model is the central composite design $[22,26]$. The detailed description about the design of experiment is not within the scope of this study and readers may refer to the relevant literature $[22,26]$. Lastly, it is noted that in this study RS models are used in the inverse optimization stage for parameter prediction.

\subsection{Uncertainty significance evaluation}

Parameter selection is a precondition for model updating and the response must be sensitive to parameter variations. Traditional experiential judgment is unreliable for complex structures and parameter sensitivity analysis often requires small parameter perturbations. Alternatively, the analysis of variance is a more efficient and reliable method, in which parameter variance analysis are used for significance evaluation. In ANOVA, the total variance of a group of parameter samples is decomposed into two parts: variance due to the random error resulting from measurement uncertainty or other sources, and variance due to parameter effects. The latter variance type embodies parameter significance and in practice, the comparison between two types of variances can be done based on the F-test evaluation $[22,26]$ :

$$
F_{x}=\frac{S S_{x} / d_{x}}{S S_{e} / d_{e}} \sim F\left(d_{x}, d_{e}\right)
$$

where $F_{x}$ and $F\left(d_{x}, d_{e}\right)$ denote the $F$-test value of parameter $x$ and the chosen criterion; $S S_{x}$ and $S S_{e}$ denote the sums of square (namely the variance) of $x$ and the random error $e$, respectively; $d_{x}$ and $d_{e}$ represent the degrees of freedom of $x$ and $e$ in the ANOVA model respectively. A result of $F_{x}>F\left(d_{x}, d_{e}\right)$ indicates that the parameter effect has significant contribution to the total model variance and thus $x$ is a significant parameter that should be considered in updating. Practically, the ANOVA is implemented using $2^{k}$ factorial designs [22,26], which is particularly useful for parameter screening purposes [6,7]. In this design, each parameter has only two levels of \pm 1 representing the upper $(+1)$ and lower $(-1)$ bounds of the parameter. In a deterministic significance evaluation, the bounds of all parameters are set to be equal for fair evaluation, e.g. $\pm 5 \%$ change with respect to the parameter initial values. But for uncertainty significance evaluation (USE) considering variability influence, the parameter bounds are set to be their variability ranges and thus not equal. The application of USE is demonstrated in the examples of this study.

\subsection{Monte Carlo simulation}

MC methods $[27,28]$ have been widely applied to the solutions of different mathematical and statistical problems. The fundamental idea of MCS consists in seeking the occurrence frequency of an event or the statistical expectation of a variable by means of stochastic sampling experiments. The advantages of MC methods (or say MCS) lie in their easy implementation, satisfactory estimation accuracy and convergence speed independent of problem dimensions, etc. On the contrary, the primary drawback is referred to the huge computational costs for most practical problems. It is noted that the approximation accuracy of MCS can be improved by having more simulation samples. Sawilowsky [29] summarizes 
characteristics of a high-quality MCS such as (a) the pseudo-random number generator has a long "period" before repeating values and produces values that pass tests for randomness; (b) the repetitions of the experiment are sufficient to ensure the accuracy and (c) the proper sampling technique is adopted. In this analysis, MCS is adopted for generating response samples from the assumed or measured probability distributions of responses.

\section{Stochastic model updating}

A model updating process involving uncertainty analysis is defined as SMU. The parameters and responses are firstly supposed to follow certain probability distributions. By establishing an inverse problem, the probability distributions of parameters can be predicted and expressed by statistical properties such as means and variances (standard deviations as well). The objective function is formed containing the statistical properties of the analytical and measured responses and then is minimized using optimization algorithms. Compared with deterministic model updating, an SMU problem is generally much more complicated and difficult to describe in a mathematical way. Moreover, considerable computational cost is required since (a) in each iteration, a forward propagation of parameter variability must be performed first to estimate the probability distributions of the response; (b) the gradient computation in the subsequent inverse optimization process becomes very difficult and time-consuming considering the involvement of uncertainty analysis. And worse, the optimization problem is often ill-conditioned inducing solution difficulty. Hence, the simplification of an SMU process is always preferred without the price of losing prediction accuracy, as has been done in this study.

\subsection{Deterministic decomposition of a stochastic model updating problem}

To simplify an SMU problem, this study attempts to decompose an SMU process into a series of deterministic ones without breaking the essence of the problem. The forward uncertainty propagation is then omitted and only the inverse propagation and optimization are performed. By these means, an SMU problem can be highly simplified resulting in fast computation and high cost-efficiency. It should be mentioned that the forward uncertainty propagation is usually presented in the existing methods [13-17]. But in the proposed method, the forward propagation is unnecessary and the probabilistic properties of a parameter are directly determined by the measured response under the assumption of no pure measurement uncertainty. And the parameters and responses are supposed to follow normal probability distributions. This consideration is based on the fact that for most engineering problems, a normal-distribution supposition of parameter or response uncertainties is acceptable since such distributions have been found in reality. Furthermore, even though a distribution does not follow the normal type, it can be transformed into a normal one by using statistical transformation methods [15].

To be specific, firstly, the USE is performed for identifying significant parameters. Then based on the repetitive response measurements of a single structure or a set of nominally identical structures, the probability distributions of the responses are estimated and expressed by means and variances for a normal distribution. The following step consists in applying MCS to the response distribution in order to generate sufficient numerical samples. This analysis employs the function randn offered by Matlab ${ }^{\circledR}$ [30] for generating pseudorandom numbers drawn from the standard normal distribution. At the meantime, RS models are constructed to replace original FE models for fast computation and optimization since a response surface is actually a mathematical expression, the establishment of an optimization problem becomes much easier and faster than that in the traditional FE model updating problems. Subsequently, with respect to each sample, a deterministic inverse problem is formed and the optimization is performed to identify the corresponding parameter values. This step is repeated until the last sample and finally numerous parameter values are obtained. Herein the gradient computation is implemented using the RS model actually being a mathematical expression providing a fast and costefficient optimization process. Lastly, the parameter means and variances can be statistically estimated based on the predicted values. The application flowchart of this method is illustrated in Fig. 1.

It is clarified that in practice, it's impossible to test structures for thousands of times in order to obtain adequate experimental samples. Therefore for stochastic updating, one has to artificially generate numerous samples using MCS according to response distributions estimated from the limited test measurements. And due to the utilization of MCS, the estimated mean and variance of a parameter will converge to stable values (or an interval) once the samples are sufficient. Furthermore, the efficiency of the proposed method also lies in its highly reducing the programming difficulty which is always headachy for the present stochastic model updating problems updating the means and variances in a single optimization.

\subsection{Optimization algorithm}

The structural frequency has been chosen as the response in all the examples of this study. A single objective function $F(x)$ contains the dimensionless expression of a frequency pair:

$$
F(x)=a b s\left(\frac{f_{\mathrm{RSM}}-f_{\mathrm{MCS}}}{f_{\mathrm{MCS}}}\right)
$$




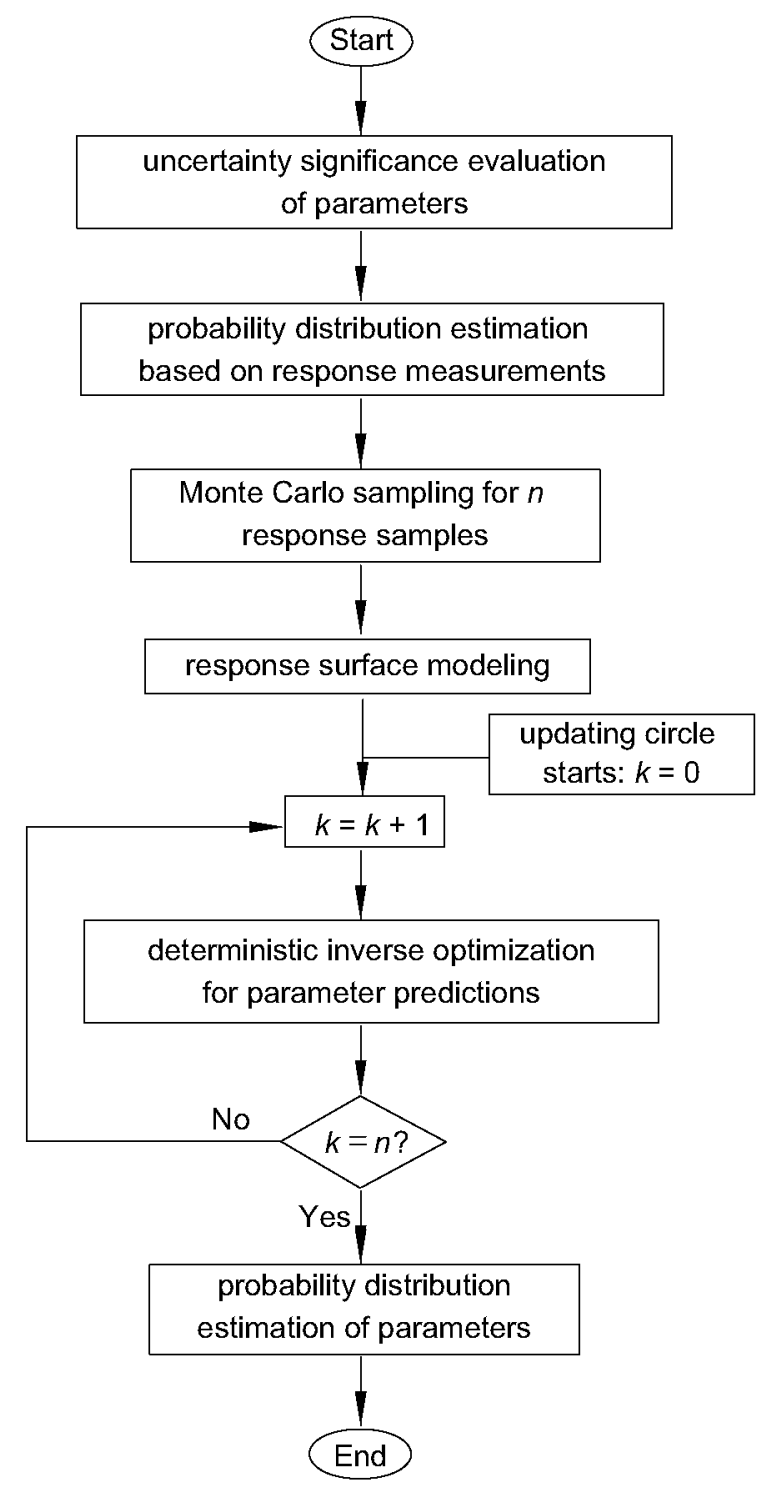

Fig. 1. Flowchart of the stochastic model updating method.

where $f_{\text {RSM }}$ and $f_{\text {MCS }}$ denote the certain-order frequency predicted by the RS model and obtained by using MCS (samples), respectively; $x$ stands for a parameter. The dimensionless employment guarantees equal updating of each objective function. Then the updating is implemented by using a multiobjective optimization algorithm:

$$
\min _{x, \gamma} \gamma,\left\{\begin{array}{l}
F(x)-\omega \cdot \gamma \leq \text { goal } \\
x \leq x \leq \bar{x}
\end{array}\right.
$$

where the weight $\omega$ controls the relative under- or over-attainment of all the objectives trying to attain the goal and $\gamma$ is a slack variable used as a dummy argument to minimize $F(x) ; \underline{x}$ and $\bar{x}$ are the lower and upper bounds of $x$. In this study the multiobjective optimization algorithm fgoalattain from Matlab ${ }^{\circledR}$ [30] has been employed, which uses a sequential quadratic programming method.

\section{Case study I: A numerical beam}

The proposed method is first validated by a numerical simply-supported beam as shown in Fig. 2 . The beam is divided into 30 identical finite elements with the cross-section dimension of $25 \times 25 \mathrm{~cm}$, the elastic modulus of $30 \mathrm{GPa}$, the density of $2400 \mathrm{~kg} / \mathrm{m}^{3}$ and Poisson ratio of 0.2 .

\subsection{Uncertainty significance evaluation}

The first four flexural frequencies of the beam were adopted as the responses. Four parameters of the elastic modulus $(E)$, the section inertia $(I)$, the density $(D)$ and Poisson ratio $(P)$ have been investigated for their significance to the response 


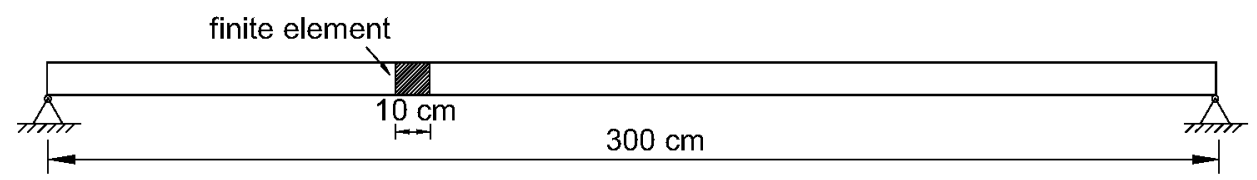

Fig. 2. A numerical simply-supported beam.
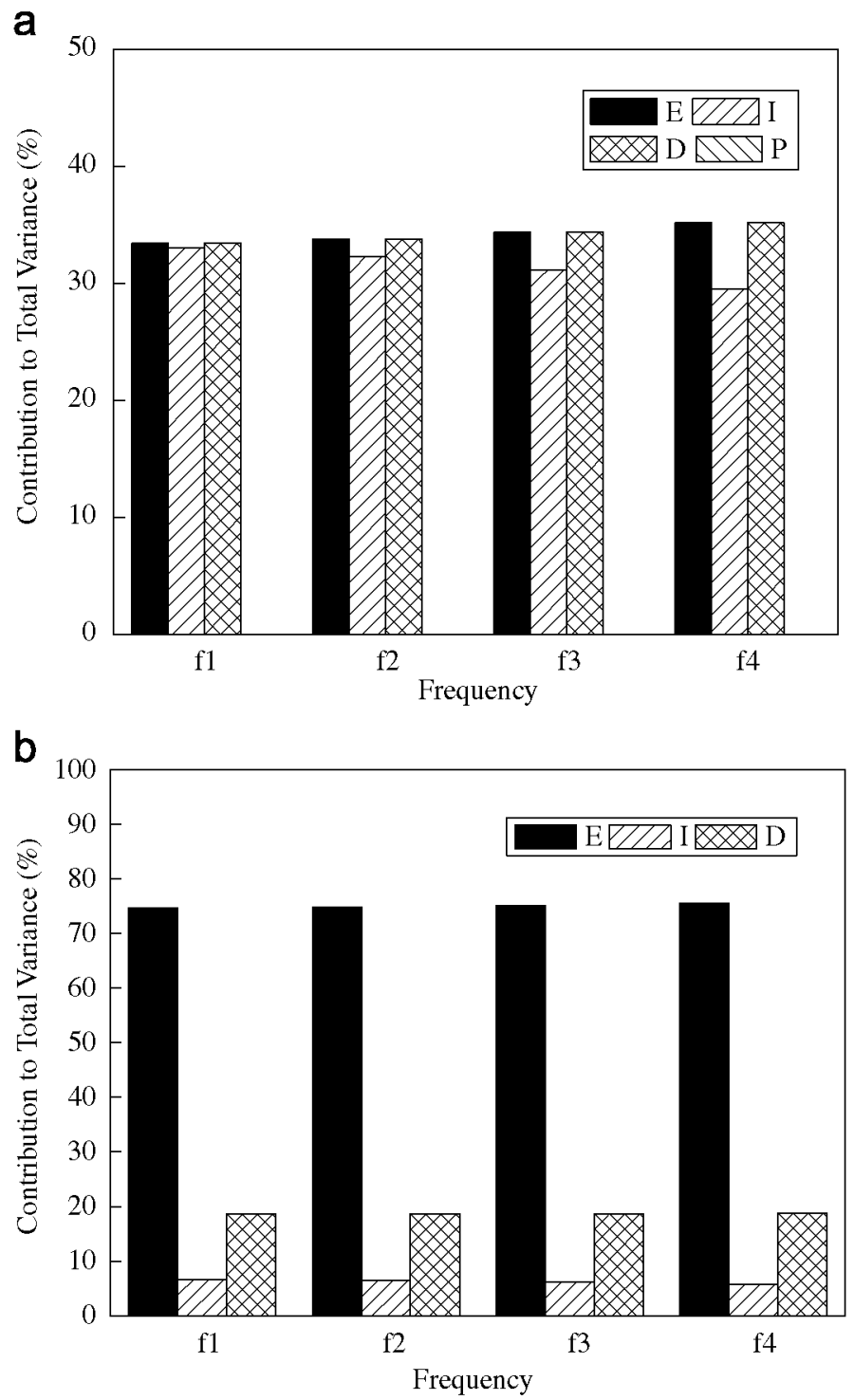

Fig. 3. (a) Deterministic significance and (b) uncertainty significance evaluations of beam parameters.

by using the ANOVA described in Section 2.2. The first-stage significance evaluation was deterministic where the frequency sensitivity to the parameter variations had to be explored for the sake of discarding non-significant parameters and thus simplifying the subsequent analysis. Here the identical bounds of $\pm 5 \%$ change to the initial value were assigned to each parameter in order to equally evaluate their significance. It is observed from Fig. 3(a) that without considering variability, $E, I$ and $D$ show similar significance to the four frequencies. However, all the frequencies are not sensitive to the change of $P$. This observation agrees well with the theoretical expression for the flexural frequencies of a beam, namely $\operatorname{det}\left|[K]-\omega^{2}[M]\right|=0$, in which $[K]$ and $[M]$ represent the stiffness matrix and the mass matrix, respectively; "det" denotes the determinant and $\omega$ denotes the circular frequency in $\mathrm{rad} / \mathrm{s}$. For the flexural frequencies, $[K]$ contains $E I$ and $[M]$ is related to the density $D$. $E, I$ and $D$ possess equal weights in the expression indicating their identical significance. And $P$ has no effect on the flexural frequencies because of its disappearance in the expression. Meanwhile, in Fig. 3(a) the significance level of $I$ slightly decreases for higher modes indicating that the geometric property effect on the flexural frequencies decreases with the increase of the mode number. As a conclusion, the parameter significance is quantitatively analyzed using the ANOVA and rational judgment is achieved. 
With respect to the USE, the variability (namely the bounds) of $E$ was defined as $\pm 10 \%$ while that of $I$ was assumed to be $\pm 3 \%$ taking into account the fact that practically the measurement of geometric dimensions is more accurate than material properties. At the same time the variability of $D$ remained as $\pm 5 \%$ and $P$ was discarded in this step according to the deterministic evaluation results. Fig. 3(b) demonstrates that after considering the variability influence, the significance of $E$ sharply increases up to around $75 \%$ for all the four frequencies, while that of $D$ is only around $19 \%$ at this moment. Meanwhile, the significance of $I$ decreases to around $6.5 \%$. The variability proportions among $E, I$ and $D$ are $3.3(E / I)$ and 2 $(E / D)$ respectively, whereas the significance proportions change to $11.5(E / I)$ and $4(E / D)$. The latter proportions are nearly the square of the former ones proving the nonlinear relationship between the variability and significance. This observation reveals the fact that in this example, the parameters have identical weights in the frequency expression, their variability contributions to the significance will be magnified nearly in a square proportion. However, it should also be noted that for complex structures, an explicit relationship between the parameter and response is often not available or difficult to obtain. At this moment, the ANOVA is very useful for exploring such relationship.

In this numerical example, the USE based on the ANOVA has been well demonstrated presenting quantitative evaluation of the parameter variability effects. The same procedure can be applied to more complex structures.

\subsection{Stochastic model updating for variability quantification}

In this subsection, the variability of $E, I$ and $D$ are identified using the proposed SMU method. The parameters were assumed to have normal probability distributions whose means and variances (standard deviations as well) are unknown and to be predicted. The frequency variations caused by the parameter variability also followed normal distributions whose means $\left(\mu_{f}\right)$ were 44.4, 176.1, 390.9 and $682.1 \mathrm{~Hz}$ computed using the parameter reference values. Simultaneously, the standard deviation $\sigma_{f}$ was artificially defined as $\sigma_{f}=0.05 \mu_{f}$ because of no real measurement data in this numerical example. However in practice, $\mu_{f}$ and $\sigma_{f}$ can be obtained by repetitive measurements on a single structure or a set of nominally identical structures.

MCS was applied to the frequency probability distributions and different sample numbers up to $10^{5}$ were generated for tests. Then with respect to the four frequencies, four second-order polynomial RS models were constructed using the central composite design in order to correlate the parameters with the responses. Four objective functions were subsequently established and the inverse optimization process was carried out using the multiobjective algorithm described in Section 3.2. Since the entire SMU procedure was decomposed into a series of deterministic ones (e.g. $10^{5}$ samples means $10^{5}$ deterministic updating processes), the inverse optimization were performed for $10^{5}$ times and $10^{5}$ pairs of $E, I$ and $D$ values were obtained. Finally, the means and variances (standard deviations) of these parameters were statistically estimated and listed in Tables 1 and 2.

It should be mentioned that for MCS, how many samples are sufficient for a qualified simulation is always problematic and depends on specific problems. Therefore in this example, different sample numbers were tried to find the sample number providing the stable predictions of both means and standard deviations.

\subsection{Results and discussion}

It can be observed from Table 1 that the predicted means of $E, I$ and $D$ are all very close to their reference values corresponding to the frequency means. And the scenarios having fewer than 100 samples can still present satisfactory

Table 1

Predictions of parameter means of the beam.

\begin{tabular}{rlll}
\hline Sample number & $E$ & $I$ & $D$ \\
\hline 10 & 0.9988 & 1.0000 & 0.9991 \\
50 & 1.0047 & 1.0000 & 0.9942 \\
100 & 1.0011 & 1.0000 & 0.9981 \\
200 & 0.9934 & 0.9998 & 1.0056 \\
300 & 0.9946 & 0.9999 & 1.0044 \\
400 & 0.9978 & 1.0002 & 1.0013 \\
500 & 0.9994 & 1.0000 & 0.9995 \\
1000 & 1.0009 & 0.9999 & 0.9980 \\
2000 & 0.9984 & 1.0000 & 1.0005 \\
5000 & 0.9981 & 0.9999 & 1.0008 \\
10,000 & 0.9976 & 0.9999 & 1.0013 \\
20,000 & 0.9980 & 1.0000 & 1.0009 \\
30,000 & 0.9984 & 1.0000 & 1.0005 \\
40,000 & 0.9985 & 1.0000 & 1.0005 \\
50,000 & 0.9988 & 1.0000 & 1.0002 \\
100,000 & 0.9981 & 1.0000 & 1.0008 \\
\hline
\end{tabular}

Note: The predicted parameter means have been normalized by the true values. 
Table 2

Predictions of parameter standard deviations of the beam.

\begin{tabular}{rlll}
\hline Sample number & $E$ & $I$ & $D$ \\
\hline 10 & 0.0733 & 0.0002 & 0.0739 \\
50 & 0.0557 & 0.0001 & 0.0560 \\
100 & 0.0435 & 0.0004 & 0.0434 \\
200 & 0.0477 & 0.0030 & 0.0469 \\
300 & 0.0494 & 0.0019 & 0.0486 \\
400 & 0.0468 & 0.0019 & 0.0464 \\
500 & 0.0522 & 0.0004 & 0.0521 \\
1000 & 0.0511 & 0.0019 & 0.0512 \\
2000 & 0.0501 & 0.0022 & 0.0498 \\
5000 & 0.0500 & 0.0025 & 0.0497 \\
10,000 & 0.0501 & 0.0028 & 0.0497 \\
20,000 & 0.0503 & 0.0022 & 0.0500 \\
30,000 & 0.0498 & 0.0026 & 0.0496 \\
40,000 & 0.0500 & 0.0024 & 0.0497 \\
50,000 & 0.0501 & 0.0024 & 0.0498 \\
100,000 & 0.0500 & 0.0023 & 0.0497 \\
\hline
\end{tabular}

Note: The predicted parameter standard deviations have been normalized by the means.

predictions. However, such "satisfactory" is not stable since the standard deviation predictions, which are more important and generally cannot be accurately estimated [13-15,17], vary sharply when the samples are not sufficient (Table 2). The prediction accuracy of the standard deviations becomes stable after the sample number (sn) arrives at 2000 . Therefore for this beam example, sn should be greater than 2000 for acceptable predictions of both means and standard deviations. Meanwhile for practical examples, the determination of sample numbers becomes more difficult and one method is to increase the sample number until the solution converges to a stable value (or an interval), as has been done in this analysis. Alternatively, the sample number can also be determined by analyzing statistical significance expressed by metaclouds.

It is also observed that the variability of $E$ and $D$ is both around 0.05 and is much larger than that of $I$ (around 0.0025 ). This observation is reasonable in respect to that for this simple beam, its geometric dimensions can be intuitively measured in an accurate way resulting in smaller variability. Nevertheless, the material properties such as $E$ and $D$ are practically difficult to measure with high accuracy and thus have larger variability. Another point should be clarified that in this numerical example, the uncertainty levels of the four frequencies were set identical for the demonstration of the proposed method, which is in fact impractical in real-world problems and also results in the ideal variability predictions of $E$ and $D$.

Lastly, one remark should be made that if the FE model, instead of the RS models, was used for updating, $10^{5}$ inverse optimization times would be excluded from consideration due to the immense computation needed. Even 100 runs would require a couple of hours for a normal PC configuration. But using the RS models requires only around $20 \mathrm{~s}$ for 100 runs. This advantage will be more prominent for complex structures having numerous parameters. Moreover, the establishment of well-conditioned sensitivity matrices will also be a headachy problem when using FE models for updating.

\section{Case study II: Experimental steel plates}

Thirty-three nominally identical rectangular plates were experimentally tested by Husain et al. [31] to investigate the performance of SMU on identifying parameter variability. The nominal dimensions of each plate were $564 \mathrm{~mm}$ (length) $\times 110 \mathrm{~mm}$ (width) $\times 1.45 \mathrm{~mm}$ (thickness). And the material properties were Young's modulus of $210 \mathrm{GPa}$, shear modulus of $83 \mathrm{GPa}$ and mass density of $7860 \mathrm{~kg} / \mathrm{m}^{3}$. The modal testing by an impact hammer was carried out on the plates having the free-free boundary conditions and the first five frequencies were measured with their means, variances and standard deviations listed in Table 3. It can be seen that the uncertainties of the frequency measurements are in very small magnitudes of less than $1 \%$ by the expression of the standard deviations. Detailed descriptions about the experiment can be found in Ref. [31], where the perturbation method has been used for the SMU of the plates. The geometric feature of the thickness $(t)$, as well as the material properties of Young's modulus $(E)$ and the shear modulus $(G)$, was updated in the interest of finding its mean and variance.

This study also adopted the plate experimental data for validation and the parameter variability predictions were compared with those obtained in Ref. [31]. The FE model of the plates was firstly established, as illustrated in Fig. 4. First five modes of the plates were computed and the mode shapes are also illustrated in Fig. 4.

\subsection{Uncertainty significance evaluation}

The USEs of $t$ and $t / E / G$ were conducted using the ANOVA. According to the actual measurement data of the plates, the variability of $t$ was set to be $1 \%$ and that of $E$ and $G$ was defined as $2 \%$ considering the fact that the variability of material 
Table 3

Statistical features of the plate frequencies in $\mathrm{Hz}$ (data from Ref. [31]).

\begin{tabular}{lcccrr}
\hline Frequency & 1 & 2 & 3 & \multicolumn{2}{c}{4} \\
\hline Mean & 24.12 & 66.92 & 77.65 & 131.97 & 158.80 \\
Standard deviation & 0.113 & 0.252 & 0.571 & 0.424 & 0.974 \\
Variance & 0.013 & 0.063 & 0.326 & 0.180 & 0.949 \\
\hline
\end{tabular}

Note: the decimal parts of standard deviations and variances have three figures, while those in Ref. [31] have only two.

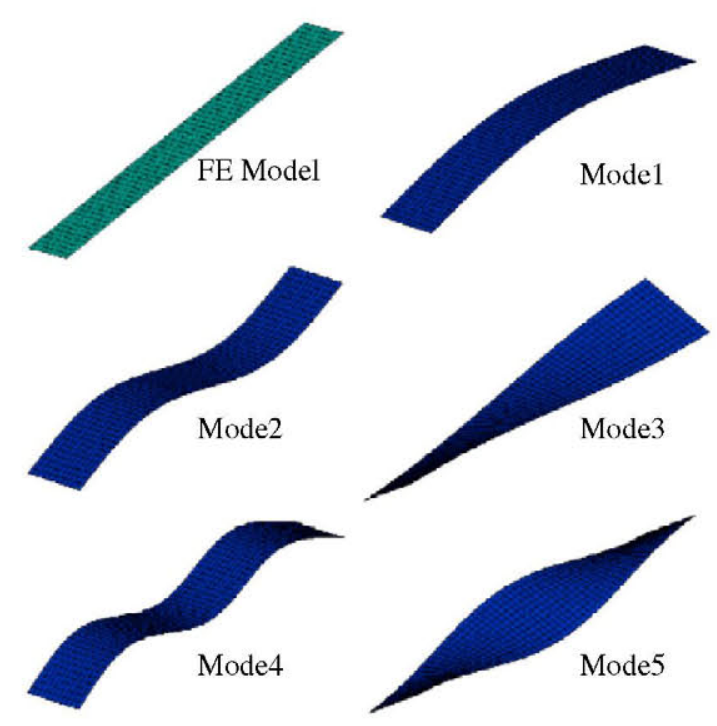

Fig. 4. The FE model and mode shapes of the plates.

properties is generally more difficult to measure. For the USE of thicknesses alone, the FE model was divided into three portions ( $t 1, t 2$ and $t 3$ ) having equal widths. Fig. 5(a) gives the evaluation results and it is observed that for the first flexural and torsional modes (modes 1 and 3), the middle plate portion (t2) is in a dominant position since this portion controls the vibrational deformation of the entire plate while the other two side portions ( $t 1$ and $t 3$ ) give little contributions. However, for the other 3 modes (modes 2, 4 and 5), the significance of t2 sharply decreases while that of $\mathrm{t} 1$ and $\mathrm{t} 3$ increases fast. Especially for mode 5 in which the side portions dominate the plate vibration, $t 2$ shows almost no effect. But for the second flexural mode, the significance of all three parameters is similar. It is noted that the significance of $\mathrm{t} 1$ and $\mathrm{t} 3$ is identical for all the modes, which proves the USE validity since the two side portions are actually symmetric and identical.

Then the USEs of the geometric and material properties of the entire plate $(t, E$ and $G)$ were conducted in the interest of observing the uncertainty effects of different types of physical quantities. Fig. 5(b) demonstrates that for the three flexural modes, $t$ and $E$ dominate the plate vibration and the significance of $E$ is higher than that of $t$. At this moment, $G$ has almost no contribution in respect that the shear modulus little affects the flexural vibrations of the plates. But for the two torsional modes, the contribution from $E$ almost disappears and $G$ turns to be the contributor. Above observations agree well with the vibration principle of plate structures. Furthermore, it is mentioned that if $t, E$ and $G$ had the same variability levels, the significance of $t$ would increase a lot with the significance reduction of $E$ and $G$. Therefore for real-world structures, once the variability of different physical quantities is not evaluated and analyzed in a correct way, the misjudgment on significant parameters is easy to occur leading to the failure of subsequent analysis.

\subsection{Stochastic model updating of thicknesses}

The thickness variability was firstly investigated and the FE model is divided into three portions ( $t 1, t 2, t 3)$ having equal widths. Based on the 33 experimental measurements [31], the statistical properties of the means and standard deviations of the five frequencies can be estimated and listed in Table 3. Then according to the frequency statistical properties, MCS was performed to generate the numerical frequency samples. Considering the investigation results of the previous beam example, a number of 2000 samples were regarded as sufficient for a stable prediction of the thickness variability. Five RS models corresponding to the five frequencies were constructed and the deterministic inverse optimization was repeated for 2000 times to obtain 2000 predicted values for each parameter. Subsequently, the parameter means $(\mu)$ and variances $\left(\sigma^{2}\right)$ were statistically estimated based on the predicted values as given in Table 4a. Compared with the results in Ref. [31], 

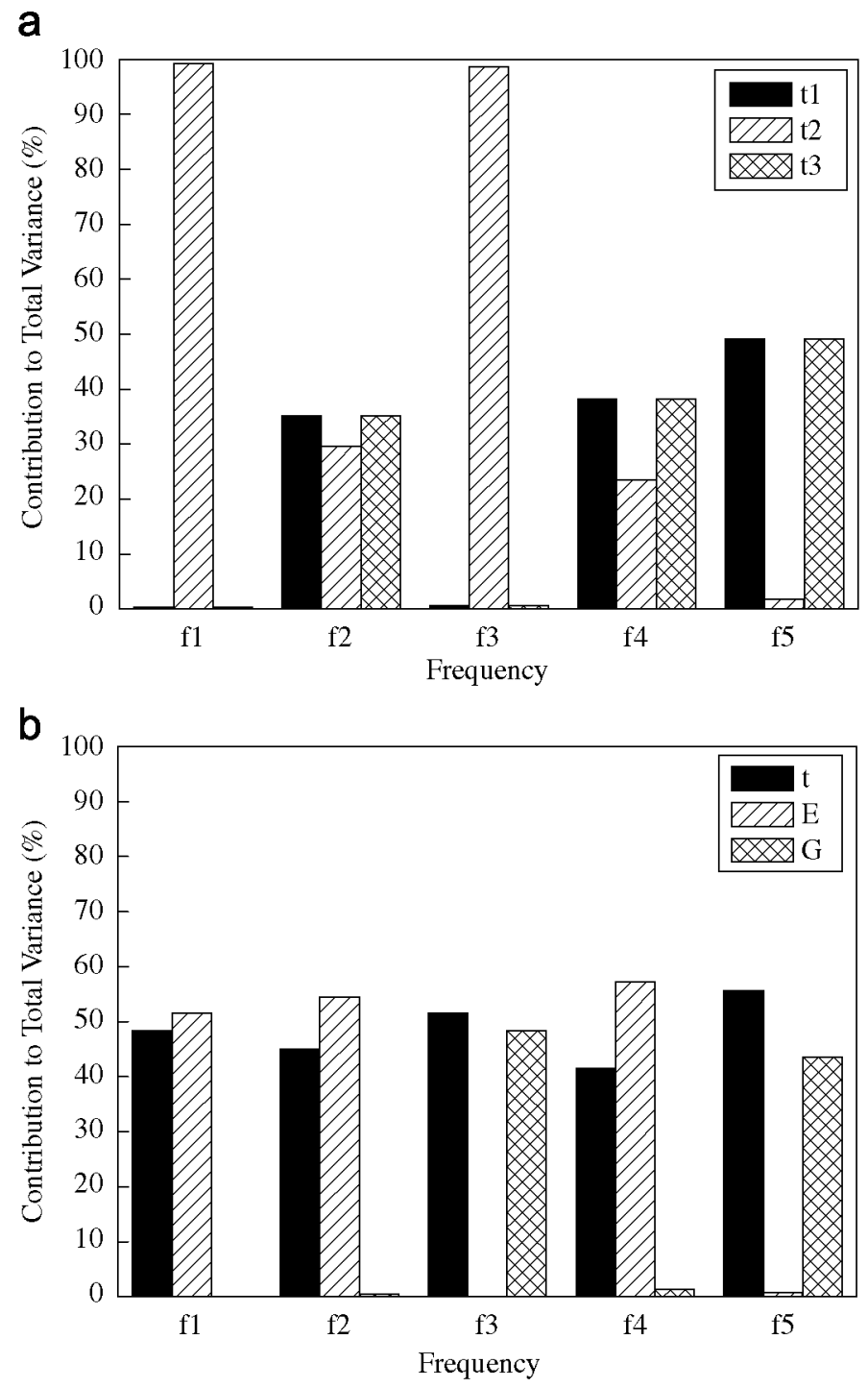

Fig. 5. Uncertainty significance evaluation of plate parameters.

Table 4a

Variability quantification results of the thicknesses in $\mathrm{mm}$.

\begin{tabular}{|c|c|c|c|c|c|c|}
\hline \multirow[t]{2}{*}{ Prediction } & \multicolumn{3}{|c|}{ Husain et al. [31] } & \multicolumn{3}{|c|}{ Proposed method } \\
\hline & $\mathrm{t} 1$ & $\mathrm{t} 2$ & $\mathrm{t} 3$ & $\mathrm{t} 1$ & $\mathrm{t} 2$ & $\mathrm{t} 3$ \\
\hline Mean & 1.4528 & 1.4493 & 1.4528 & 1.4605 & 1.4552 & 1.4605 \\
\hline Variance $\left(\times 10^{-4}\right)$ & 1.290 & 0.535 & 1.290 & 0.627 & 0.770 & 0.627 \\
\hline
\end{tabular}

Table 4b

Variability quantification results of the material properties in GPa.

\begin{tabular}{|c|c|c|c|c|}
\hline \multirow[t]{2}{*}{ Prediction } & \multicolumn{2}{|c|}{ Husain et al. [31] } & \multicolumn{2}{|c|}{ Proposed method } \\
\hline & $E$ & $G$ & $E$ & $G$ \\
\hline Mean & 209.6 & 83.8 & 209.2 & 84.9 \\
\hline Variance $\left(\times 10^{-4}\right)$ & 2.6 & 1.5 & 4.6 & 1.2 \\
\hline
\end{tabular}

the estimations by the proposed method are also symmetric, namely $\mu_{t 1}=\mu_{t 3}$ and $\sigma_{t 1}^{2}=\sigma_{t 3}^{2}$, which is reasonable since the two side portions of the plate are symmetric. The means predicted by both methods are similar while the variances have relatively large difference. The variances predicted by the proposed method seem more rational since taking into account 
Table 4c

Comparison of variability quantification results by the proposed method.

\begin{tabular}{|c|c|c|c|c|c|c|}
\hline \multirow[t]{2}{*}{ Prediction } & \multicolumn{3}{|l|}{ Separation } & \multicolumn{3}{|l|}{ Combination } \\
\hline & $t$ & $E$ & G & $t$ & $E$ & G \\
\hline Mean & 1.4587 & 209.2 & 84.9 & 1.456 & 207.23 & 84.26 \\
\hline Variance & $0.675 \times 10^{-4}$ & 4.6 & 1.2 & $0.565 \times 10^{-4}$ & 0.166 & 0.139 \\
\hline
\end{tabular}

Note: (1) the units of $\mathrm{t}$ and $\mathrm{E} / \mathrm{G}$ are $\mathrm{mm}$ and GPa, respectively; (2) the values of $\mathrm{t}$ are actually the average of $\mathrm{t} 1, \mathrm{t} 2$ and $\mathrm{t} 3$ in Table $4 \mathrm{a}$.

Table 5 a

Measured, initial and updated mean frequencies in $\mathrm{Hz}$ of the plates using thicknesses as parameters.

\begin{tabular}{|c|c|c|c|c|c|}
\hline Mode & Measured & Initial & Error & Updated & Error \\
\hline 1 & 24.12 & $24.26(24.27)$ & $0.58(0.64)$ & $24.36(24.27)$ & $1.00(0.61)$ \\
\hline 2 & 66.92 & $67.15(67.24)$ & $0.34(0.48)$ & $67.56(67.32)$ & $0.96(0.60)$ \\
\hline 3 & 77.65 & $76.56(75.31)$ & $1.40(3.01)$ & $76.88(75.29)$ & $0.99(3.03)$ \\
\hline 4 & 131.97 & $132.17(132.51)$ & $0.15(0.41)$ & $132.99(132.68)$ & $0.77(0.53)$ \\
\hline 5 & 158.80 & $156.74(154.31)$ & $1.30(2.83)$ & $157.83(154.57)$ & $0.61(2.66)$ \\
\hline Average error & & & $0.75(1.47)$ & & $0.87(1.49)$ \\
\hline
\end{tabular}

Note: the errors are in \%; the data in the parentheses are from Ref. [31] and given here for comparison.

Table 5b

Measured, initial and updated mean frequencies in $\mathrm{Hz}$ of the plates using material properties as parameters.

\begin{tabular}{|c|c|c|c|c|c|}
\hline Mode & Measured & Initial & Error & Updated & Error \\
\hline 1 & 24.12 & $24.26(24.27)$ & $0.58(0.64)$ & $24.21(24.23)$ & $0.37(0.47)$ \\
\hline 2 & 66.92 & $67.15(67.24)$ & $0.34(0.48)$ & $66.93(67.04)$ & $0.01(0.18)$ \\
\hline 3 & 77.65 & $76.56(75.31)$ & $1.40(3.01)$ & $77.41(76.67)$ & $0.31(1.25)$ \\
\hline 4 & 131.97 & $132.17(132.51)$ & $0.15(0.41)$ & $131.62(131.93)$ & $0.27(0.03)$ \\
\hline 5 & 158.80 & $156.74(154.31)$ & $1.30(2.83)$ & $158.31(156.86)$ & $0.31(1.22)$ \\
\hline Average error & & & $0.75(1.47)$ & & $0.25(0.63)$ \\
\hline
\end{tabular}

Note: the errors are in \%; the data in the parentheses are from Ref. [31] and given here for comparison.

Table 5c

Comparison of mean frequencies in $\mathrm{Hz}$ of the plates.

\begin{tabular}{|c|c|c|c|c|c|c|c|}
\hline Mode & Measured & $\mathrm{t} 1 / \mathrm{t} 2 / \mathrm{t} 3$ & Error & $E / G$ & Error & $t / E / G$ & Error \\
\hline 1 & 24.12 & 24.36 & 1.00 & 24.21 & 0.37 & 24.20 & 0.33 \\
\hline 2 & 66.92 & 67.56 & 0.96 & 66.93 & 0.01 & 66.90 & 0.03 \\
\hline 3 & 77.65 & 76.88 & 0.99 & 77.41 & 0.31 & 77.44 & 0.27 \\
\hline 4 & 131.97 & 132.99 & 0.77 & 131.62 & 0.27 & 131.55 & 0.32 \\
\hline 5 & 158.80 & 157.83 & 0.61 & 158.31 & 0.31 & 158.35 & 0.28 \\
\hline Average error & & & 0.87 & & 0.25 & & 0.25 \\
\hline
\end{tabular}

Note: the errors are in \%.

such small plates, the variability of three portions should be close. As to the predicted frequency means listed in Table 5a , it can be seen that the initial FE model established in this study is more accurate than that in Ref. [31] in the aspect of frequency prediction. Both FE models are accurate enough for subsequent analysis. After SMU, the mean errors almost remain unchanged for both methods implying the accurate and stable perditions. Then the focus comes to the frequency variance predictions (Table 6a), where both methods give unsatisfactory results (Tables 6a). Compared with Ref. [31], the proposed method presents worse predictions of the flexural frequencies but better predicts the torsional frequencies. As mentioned previously, parameter variance prediction is a pending problem for existing SMU methods. Here the unsatisfactory predictions of the frequency variances reflect the unsatisfactory performance of using the thickness parameters. Fig. 6 depicts the scatter plots of the measured and predicted frequencies after SMU. It is observed from Fig. 6(a) that the predicted frequencies failed to converge to the measured ones, as has also been found in Ref. [31]. Hence 
Table 6a

Measured and predicted frequency variances using thicknesses as parameters.

\begin{tabular}{lllllr}
\hline Mode & Measured & Husain et al. [31] & Error (\%) & Proposed method & Error (\%) \\
\hline 1 & 0.013 & 0.01 & 23.1 & 0.021 & 61.5 \\
2 & 0.063 & 0.06 & 4.8 & 0.141 & 123.8 \\
3 & 0.326 & 0.13 & 60.1 & 0.207 & 36.5 \\
4 & 0.180 & 0.24 & 33.3 & 0.544 & 202.2 \\
5 & 0.949 & 0.28 & 70.5 & 0.742 & 21.8 \\
\hline
\end{tabular}

Table 6b

Measured and predicted frequency variances using material properties as parameters.

\begin{tabular}{lllcrr}
\hline Mode & Measured & Husain et al. [31] & Error (\%) & Proposed method & Error (\%) \\
\hline 1 & 0.013 & 0.01 & 23.1 & 0.015 & 15.4 \\
2 & 0.063 & 0.06 & 4.8 & 0.114 & 81.0 \\
3 & 0.326 & 0.28 & 14.1 & 0.239 & 26.7 \\
4 & 0.180 & 0.22 & 22.2 & 0.437 & 142.8 \\
5 & 0.949 & 1.09 & 14.9 & 0.957 & 0.8 \\
\hline
\end{tabular}

Table 6c

Comparison of frequency variances of the plates.

\begin{tabular}{lllrrrrr}
\hline Mode & Measured & $\mathrm{t} 1 / \mathrm{t} 2 / \mathrm{t} 3$ & \multicolumn{1}{c}{ Error } & $\mathrm{E} / \mathrm{G}$ & Error & $t / E / G$ & Error \\
\hline 1 & 0.013 & 0.021 & 61.5 & 0.015 & 15.4 & 0.010 & 23.1 \\
2 & 0.063 & 0.141 & 123.8 & 0.114 & 81.0 & 0.068 & 7.9 \\
3 & 0.326 & 0.207 & 36.5 & 0.239 & 26.7 & 0.318 & 2.5 \\
4 & 0.180 & 0.544 & 202.2 & 0.437 & 142.8 & 0.238 & 32.2 \\
5 & 0.949 & 0.742 & 21.8 & 0.957 & 0.8 & 1.234 & 30.0 \\
\hline
\end{tabular}

at this moment the thickness variability predictions are not reliable. Other types of parameters should be adopted for stochastic updating.

\subsection{Stochastic model updating of Young's and shear moduli}

As is investigated in Ref. [31], the material properties of Young's modulus and the shear modulus were also stochastically updated in this analysis. The predicted parameter means and variances are given in Table $4 \mathrm{~b}$ demonstrating that the predictions are very close to those obtained by Husain et al. [31], except the variances of $E$ having relatively larger difference. Meanwhile, compared with the results in the previous subsection, the updated frequencies are more accurate with an improvement in the mean error from 0.87 to 0.25 (Table 5b). Similar improvement is also found in Ref. [31]. Then concerning the frequency variances embodying the parameter variability, considerable improvement in the prediction accuracy has been found in Table 6b. And in this case, the first flexural frequency prediction is better than that in Ref. [31]. At the same time, Fig. 6(b) illustrates that the predicted frequencies well fit the measured ones indicating satisfactory convergence. Therefore, a conclusion can be drawn that using the material properties as the parameters for SMU may present much better performance than using the thicknesses alone, as is also reached in Ref. [31].

\subsection{Stochastic model updating on the parameter combination of geometric and material properties.}

In addition to above investigations, the thickness, Young's modulus and the shear modulus of an entire plate have also been simultaneously updated and compared with the results from the previous two subsections. Table $4 \mathrm{c}$ shows that the mean predictions of the three parameters $(t, E$ and $G)$ are very similar while the variance predictions of $E$ and $G$ are quite different. The predictions from the parameter-combination updating become very small in the magnitudes implying that the variability of $E$ and $G$ is in fact much smaller. Table $5 c$ compares the predictions of the frequency means finding that the average error from the parameter-combination updating is the same as that from Section 5.3. However, it can be seen from Table $6 \mathrm{c}$ that the frequency variance predictions are much better than the previous analysis. The predictions for both flexural and torsional modes are highly and equally improved. And by comparing Fig. 6(c) with (b), it is also observed that the frequency convergence becomes better. Hence, combining different parameter types may improve variability predictions. 

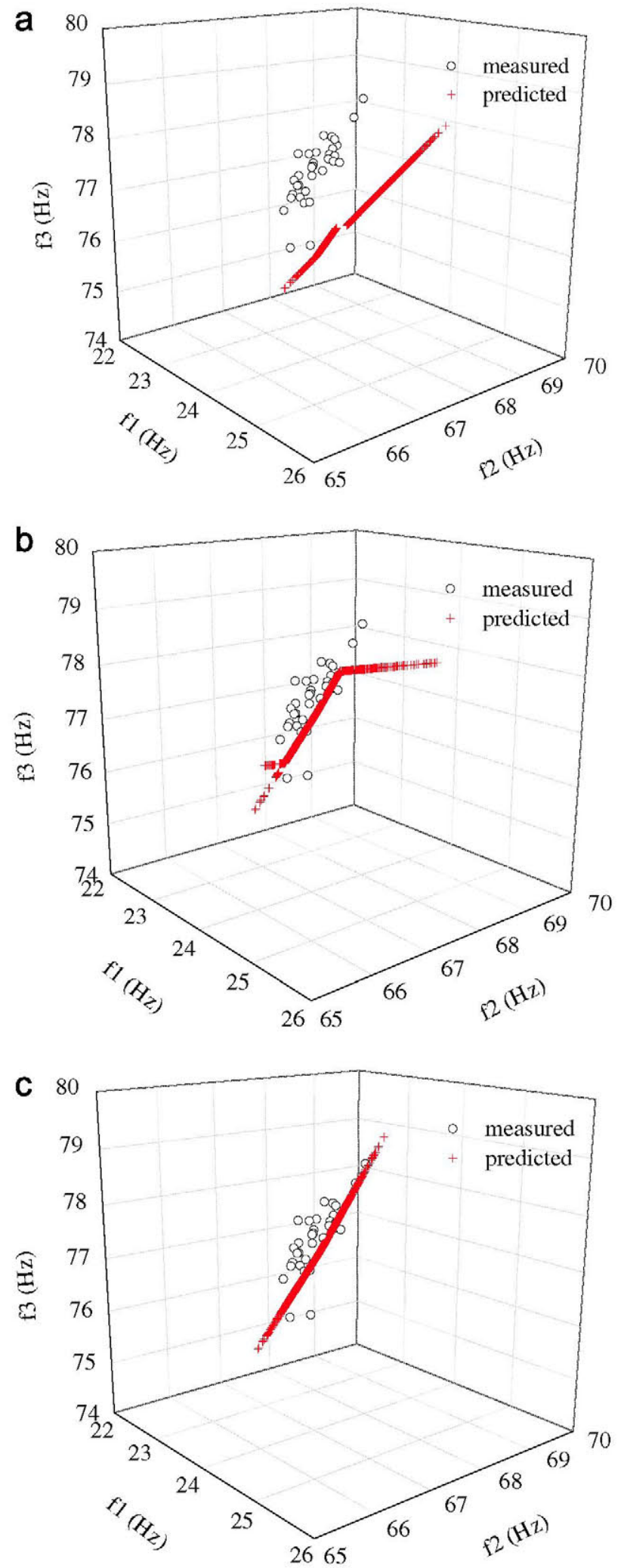

Fig. 6. Scatter plots of plate frequencies after stochastic updating (a) using thicknesses as parameters; (b) using material properties as parameters; (c) using the combination of thickness and material properties.

\section{Conclusions}

A stochastic model updating method has been developed in this paper for quantifying structural parameter variability. The idea of decomposing a stochastic updating process into a series of deterministic ones can considerably simplify the complexity of the problem and at the same time provide high cost-efficiency in computation. Response surface models are 
used as surrogates of original FE models for response computation and inverse optimization. And Monte Carlo simulation has been employed for generating samples based on the measured probability distributions of the response. The combination of response surface models and Monte Carlo simulation realizes the implementation of the proposed method, which has been validated on a numerical beam and, more convincing, on a set of experimental steel plates. The investigation results demonstrate the feasibility and cost-efficiency of the proposed method. And after comparison with an existing method, the accuracy of the proposed method has also been proved. Meanwhile, uncertainty significance evaluation of parameters with the aid of the analysis of variance method is also carried out in the study. By this means, the uncertainty effects of parameters can be quantitatively analyzed providing more reliable selection of updating parameters. On the other hand, the proposed method also has its limitations and deserves further improvement on its theory. The uncertainties in measurements are assumed to be caused only by the parameter variability. Practically, the measurement uncertainty induced by environment and devices should also be reflected in the updating process. And for complex structures having many parameters, the adoption of higher-order response surface models might be required.

\section{Acknowledgments}

The research is supported by the National Natural Science Foundation of China (Grant no. 51108090) and also by the Natural Science Foundation of Fujian Province (Grant no. 2011J05129). The foundation support from Fuzhou University (Projects 2010-XQ-26 and XRC-1048) is also acknowledged.

\section{References}

[1] M.I. Friswell, J.E. Mottershead, Finite Element Model Updating in Structural Dynamics, Kluwer Academic Publishers, Dordrecht, Netherlands, 1995.

[2] J.E. Mottershead, M. Link, M.I. Friswell, The sensitivity method in finite element model updating: a tutorial, Mech. Syst. Signal Process. 25 (7) (2011) 2275-2296.

[3] A. Teughels, J. Maeck, G. De Roeck, Damage assessment by FE model updating using damage functions, Comput. Struct. 80 (25) (2002) 1869-1879.

[4] S.E. Fang, R. Perera, G. De Roeck, Damage identification of a reinforced concrete frame by finite element model updating using damage parameterization, J. Sound Vib. 313 (3-5) (2008) 544-559.

[5] M. Link, M. Weiland, Damage identification by multi-model updating in the modal and in the time domain, Mech. Syst. Signal Process. 23 (6) (2009) $1734-1746$.

[6] S.E. Fang, R. Perera, A response surface methodology based damage identification technique, Smart Mater. Struct. 18 (6) (2009) 065009.

[7] S.E. Fang, R. Perera, Damage identification by response surface model updating using D-optimal design, Mech. Syst. Signal Process. 25 (2) (2011) 717-733.

[8] D. Moens, A Non-Probabilistic Finite Element Approach for Structural Dynamic Analysis With Uncertain Parameters, Ph.D. Thesis, Catholic University of Leuven, Belgium, 2002.

[9] J.D. Collins, G.C. Hart, T.K. Hasselman, B. Kennedy, Statistical identification of structures, AlAA J 12 (2) (1974) 185-190.

[10] M.I. Friswell, The adjustment of structural parameters using a minimum variance estimator, Mech. Syst. Signal Process. 3 (2) (1989) 143-155.

[11] J.L. Beck, L.S. Katafygiotis, Updating models and their uncertainties. I: Bayesian statistical framework, J. Eng. Mech. 124 (4) (1998) $455-461$.

[12] G. Steenackers, P. Guillaume, Finite element model updating taking into account the uncertainty on the modal parameters estimates, J. Sound Vib. $296(4-5)(2006)$ 919-934.

[13] C. Mares, J.E. Mottershead, M.I. Friswell, Stochastic model updating: part 1—theory and simulated example, Mech. Syst. Signal Process. 20 (7) (2006) $1674-1695$.

[14] C. Mares, J.E. Mottershead, M.I. Friswell, Stochastic model updating: part 2 -application to a set of physical structures, Mech. Syst. Signal Process. 20 (7) (2006) 2171-2185

[15] M.I. Friswell, J.E. Mottershead, C. Mares, Stochastic model updating in structural dynamics, in: Proceedings of the ECCOMAS Thematic Conference on Computational Methods in Structural Dynamics and Earthquake Engineering, Rethymno, Crete, Greece, 2007.

[16] X.G. Hua, Y.Q. Ni, Z.Q. Chen, J.M. Ko, An improved perturbation method for stochastic finite element model updating, Int. J. Numer. Methods Eng. 73 (2008) 1845-1864

[17] H.H. Khodaparast, J.E. Mottershead, M.I. Friswell, Perturbation methods for the estimation of parameter variability in stochastic model updating, Mech. Syst. Signal Process. 22 (8) (2008) 1751-1773.

[18] H.H. Khodaparast, J.E. Mottershead, K.J. Badcock, Propagation of structural uncertainty to linear aeroelastic stability, Comput. Struct. 88 (3-4) (2010) $223-236$.

[19] S. Adhikari, M.I. Friswell, Random matrix eigenvalue problems in structural dynamics, Int. J. Numer. Methods Eng. 69 (2007) 562-591.

[20] Y. Govers, M. Link, Stochastic model updating-covariance matrix adjustment from uncertain experimental modal data, Mech. Syst. Signal Process. 24 (3) (2010) 696-706.

[21] A. Saltelli, Sensitivity analysis for importance assessment, in: Proceedings of the 3rd International Symposium on Sensitivity Analysis of Model Output, CIEMAT Publication, Madrid, 2001.

[22] D.C. Montgomery, Design and Analysis of Experiments, sixth edition, J. Wiley \& Sons, 2004.

[23] M.J. Atalla, D.J. Inman, On model updating using neural networks, Mech. Syst. Signal Process. 12 (1) (1998) $135-161$.

[24] Q.T. Guo, L.M. Zhang, Finite element model updating based on response surface methodology, in: Proceedings of the 22nd International Modal Analysis Conference, Dearborn, Michigan, 2004.

[25] A.L. Cundy. Use of Response Surface Metamodels in Damage Identification of Dynamic Structures. M.S. Thesis. Dept. of Engineering Science and Mechanics, Virginia Polytechnic Institute and State University, 2002.

[26] R.H. Myers, D.C. Montgomery, C.M. Anderson-Cook, Response Surface Methodology: Process and Product Optimization Using Designed Experiments, 3rd edition, John Wiley \& Sons, New York, 2009.

[27] I.M. Sobol, A Primer for the Monte Carlo Method, CRC press, 1994

[28] G.S. Fishman, Monte Carlo Concepts, Algorithms, and Applications, Kluwer Academic Publishers, Springer, New York, 2003.

[29] S.S. Sawilowsky, Invited debate: target article-you think you've got trivials? J. Mod. Appl. Stat. Methods 2 (1) (2003) $218-225$

[30] Matlab ${ }^{(B)}$ Rev. 7.4, MathWorks Inc, Natick, Massachusetts, USA, 2007.

[31] N.A. Husain, H.H. Khodaparast, H.J. Ouyang, Parameter selections for stochastic uncertainty in dynamic models of simple and complicated structures, in: Proceedings of the 10th International Conference on Recent Advances in Structural Dynamics, University of Southampton, Southampton, 2010. 